INLO-PUB-12/97

hep-ph/9712209

November 1997

\title{
Threshold and pseudothreshold values of the sunset diagram
}

\author{
F.A. Berends ${ }^{a}$, 丹, A.I. Davydychev ${ }^{a, b,}$ ? and N.I. Ussyukina ${ }^{b}$, \\ ${ }^{a}$ Instituut-Lorentz, University of Leiden, \\ P.O.B. 9506, 2300 RA Leiden, The Netherlands \\ ${ }^{b}$ Institute for Nuclear Physics, Moscow State University, \\ 119899 Moscow, Russia
}

\begin{abstract}
Analytic results for the threshold and pseudothreshold values of the sunset diagram with arbitrary masses are obtained in terms of dilogarithms of ratios of the masses.
\end{abstract}

\footnotetext{
${ }^{1}$ E-mail address: berends@lorentz.leidenuniv.nl

${ }^{2}$ E-mail address: davyd@theory.npi.msu.su

${ }^{3}$ E-mail address: ussyuk@theory.npi.msu.su
} 


\section{Introduction}

We shall deal with Feynman integrals corresponding to the so-called "sunset" diagram. This is a two-loop, self-energy-type diagram involving three propagators. In what follows, we shall need different integer powers $\nu_{i}$ of the corresponding denominators. The scalar integrals corresponding to this diagram with an external momentum $k$ are denoted as

$$
L\left(n ; \nu_{1}, \nu_{2}, \nu_{3}\right) \equiv \iint \frac{\mathrm{d}^{n} p \mathrm{~d}^{n} q}{\left[(p-q)^{2}-m_{1}^{2}\right]^{\nu_{1}}\left[q^{2}-m_{2}^{2}\right]^{\nu_{2}}\left[(k-p)^{2}-m_{3}^{2}\right]^{\nu_{3}}},
$$

where $n=4-2 \varepsilon$ is the space-time dimension in the framework of dimensional regularization [1]. The sunset diagram possesses a three-particle threshold at $k^{2}=\left(m_{1}+m_{2}+m_{3}\right)^{2}$ and (in general) three pseudothresholds, at $k^{2}=\left(m_{1}+m_{2}-m_{3}\right)^{2},\left(m_{1}-m_{2}+m_{3}\right)^{2}$ and $\left(-m_{1}+m_{2}+m_{3}\right)^{2}$.

There are several reasons why analytical evaluation of such diagrams and, in particular, its threshold and peudothreshold values, is important:

(i) They are needed for calculation of some realistic radiative corrections in the Standard Model and its extensions (in particular, in the Higgs sector). The sunset-type integral is a part of the basis for any two-loop two-point calculation (see e.g. in [2]).

(ii) Although this is one of the basic two-loop-order diagrams in the Quantum Field Theory, analytic results for general values of the external momentum $k$ and the masses $m_{i}$ are not available円, at least in terms of special functions like (generalized) polylogarithms. Moreover, there are some arguments [6] that the results for such diagrams cannot be expressed in terms of polylogartihms, with a possible exception of special values of $k^{2}$.

(iii) This is the simplest example of a diagram involving a three-particle cut with all the three particles being massive. Exact results for this diagram would be useful for understanding the analytic structure of three-particle cuts. Note that the four-dimensional case is much more complicated than the three-dimensional one considered in [7].

(iv) The threshold values of such diagrams are needed for constructing analytic approximations of the behaviour near the threshold, cf. e.g. refs. [8, 9, 10].

(v) In some cases (for example, when two masses are equal, $m_{1}=m_{2}$ ), the pseudothreshold may coincide with the on-shell limit $k^{2}=m_{3}^{2}$ which is relevant for the on-shell calculations (see e.g. in refs. [11, 12]).

(vi) Some numerical approaches (in particular, the one described in [13]) involve the sunset-like integrals as "kernels" of integral representations for more general two-loop graphs. It should be noted that some integral representations which can be used for numerical calculation of two-loop self-energy diagrams can also be found in refs. [14, 15].

(vii) The threshold value corresponds to an infinite sum related to the small momentum expansion [16, 17, 18], or the large momentum expansion [19], taken at its border of convergence. The closed form for the coefficients of such expansions (in terms of generalized hypergeometric functions) was given in ref. [20]. Therefore, as a by-product of the calculation one could get some summation formulae for complicated hypergeometric functions. Furthermore, since the sunset diagram may be represented in terms of a one-dimensional integral involving four Bessel-type functions [21, 20], the analytic results are applicable to those integrals, too.

\footnotetext{
${ }^{1}$ The results for some other two-loop self-energy diagrams can be found in refs. [3, 田, 青, 6].
} 


\section{Approach to the calculation}

The standard Feynman parametric representation for the integral (1) reads

$$
\begin{aligned}
L\left(n ; \nu_{1}, \nu_{2}, \nu_{3}\right) & =\mathrm{i}^{2-2 n} \pi^{n} \frac{\Gamma\left(\nu_{1}+\nu_{2}+\nu_{3}-n\right)}{\Gamma\left(\nu_{1}\right) \Gamma\left(\nu_{2}\right) \Gamma\left(\nu_{3}\right)} \int_{0}^{1} \int_{0}^{1} \int_{0}^{1} \frac{\prod \alpha_{i}^{\nu_{i}-1} \mathrm{~d} \alpha_{i} \delta\left(\sum \alpha_{i}-1\right)}{\left(\alpha_{1} \alpha_{2}+\alpha_{1} \alpha_{3}+\alpha_{2} \alpha_{3}\right)^{3 n / 2-\Sigma \nu_{i}}} \\
& \times \frac{1}{\left[\alpha_{1} \alpha_{2} \alpha_{3} k^{2}-\left(\alpha_{1} \alpha_{2}+\alpha_{1} \alpha_{3}+\alpha_{2} \alpha_{3}\right)\left(\alpha_{1} m_{1}^{2}+\alpha_{2} m_{2}^{2}+\alpha_{3} m_{3}^{2}\right)\right]^{\Sigma \nu_{i}-n}} .
\end{aligned}
$$

Using tricks similar to those described in [6], we can get rid of the first denominator in the integrand of eq. (2). Namely, let us use exactly the same transformation of $\alpha$-variables (first inverting and then rescaling) as in eq. (13) of [22],

$$
\alpha_{i}=\left(\alpha_{i}^{\prime}\right)^{-1}, \quad \alpha_{i}^{\prime}=\mathcal{F}\left(\alpha^{\prime \prime}{ }_{1}, \alpha^{\prime \prime}{ }_{2}, \alpha^{\prime \prime}{ }_{3}\right), \quad \mathcal{F}\left(\alpha^{\prime \prime}{ }_{1}, \alpha^{\prime \prime}{ }_{2}, \alpha^{\prime \prime}{ }_{3}\right)=\frac{\alpha_{1}^{\prime \prime-1}+\alpha^{\prime \prime}{ }_{2}^{-1}+\alpha^{\prime \prime}{ }_{3}^{-1}}{\alpha^{\prime \prime}{ }_{1}+\alpha^{\prime \prime}{ }_{2}+\alpha^{\prime \prime}{ }_{3}}
$$

Suppressing the primes, we arrive at the following modified representation:

$$
\begin{aligned}
L\left(n ; \nu_{1}, \nu_{2}, \nu_{3}\right) & =\mathrm{i}^{2-2 n} \pi^{n} \frac{\Gamma\left(\nu_{1}+\nu_{2}+\nu_{3}-n\right)}{\Gamma\left(\nu_{1}\right) \Gamma\left(\nu_{2}\right) \Gamma\left(\nu_{3}\right)} \\
& \times \int_{0}^{1} \int_{0}^{1} \int_{0}^{1} \frac{\alpha_{1}^{\nu_{2}+\nu_{3}-n / 2-1} \alpha_{2}^{\nu_{1}+\nu_{3}-n / 2-1} \alpha_{3}^{\nu_{1}+\nu_{2}-n / 2-1} \prod \mathrm{d} \alpha_{i} \delta\left(\sum \alpha_{i}-1\right)}{\left[\alpha_{1} \alpha_{2} \alpha_{3} k^{2}-\alpha_{2} \alpha_{3} m_{1}^{2}-\alpha_{1} \alpha_{3} m_{2}^{2}-\alpha_{1} \alpha_{2} m_{3}^{2}\right]^{\Sigma \nu_{i}-n}} .
\end{aligned}
$$

The equivalence of the representations (2) and (4) can also be established by comparing the corresponding triple Mellin-Barnes contour integrals.

Furthermore, using Cheng-Wu theorem [23] (see also in [24], Appendix B) and rescaling the variables, one can transform the representation (14) into

$$
\begin{aligned}
& L\left(n ; \nu_{1}, \nu_{2}, \nu_{3}\right)=\mathrm{i}^{2-2 \Sigma \nu_{i}} \pi^{n} \frac{\Gamma\left(\nu_{1}+\nu_{2}+\nu_{3}-n\right)}{\Gamma\left(\nu_{1}\right) \Gamma\left(\nu_{2}\right) \Gamma\left(\nu_{3}\right)}\left(\prod m_{i}^{n / 2-\nu_{i}}\right) \\
& \times \int_{0}^{\infty} \int_{0}^{\infty} \frac{\mathrm{d} \xi \mathrm{d} \eta \xi^{\nu_{2}+\nu_{3}-n / 2-1} \eta^{\nu_{1}+\nu_{3}-n / 2-1}}{\left(m_{1} \xi+m_{2} \eta+m_{3}\right)^{3 n / 2-\Sigma \nu_{i}}\left[\left(m_{1} \xi+m_{2} \eta+m_{3}\right)\left(m_{1} \eta+m_{2} \xi+m_{3} \xi \eta\right)-k^{2} \xi \eta\right]^{\Sigma \nu_{i}-n}} .
\end{aligned}
$$

The cubic form in the denominator has the following representations at the pseudothresholds and at the threshold:

$$
\begin{aligned}
& {\left[\left(m_{1} \xi+m_{2} \eta+m_{3}\right)\left(m_{1} \eta+m_{2} \xi+m_{3} \xi \eta\right)-k^{2} \xi \eta\right]} \\
& =m_{2} m_{3} \xi(1-\eta)^{2}+m_{1} m_{3} \eta(1-\xi)^{2}+m_{1} m_{2}(\xi-\eta)^{2}+\left(\left(m_{1}+m_{2}+m_{3}\right)^{2}-k^{2}\right) \xi \eta \\
& = \begin{cases}m_{2} m_{3} \xi(1-\eta)^{2}+m_{1} m_{3} \eta(1+\xi)^{2}+m_{1} m_{2}(\xi+\eta)^{2}, & k^{2}=\left(-m_{1}+m_{2}+m_{3}\right)^{2} \\
m_{2} m_{3} \xi(1+\eta)^{2}+m_{1} m_{3} \eta(1-\xi)^{2}+m_{1} m_{2}(\xi+\eta)^{2}, & k^{2}=\left(m_{1}-m_{2}+m_{3}\right)^{2} \\
m_{2} m_{3} \xi(1+\eta)^{2}+m_{1} m_{3} \eta(1+\xi)^{2}+m_{1} m_{2}(\xi-\eta)^{2}, & k^{2}=\left(m_{1}+m_{2}-m_{3}\right)^{2} \\
m_{2} m_{3} \xi(1-\eta)^{2}+m_{1} m_{3} \eta(1-\xi)^{2}+m_{1} m_{2}(\xi-\eta)^{2}, & k^{2}=\left(m_{1}+m_{2}+m_{3}\right)^{2}\end{cases}
\end{aligned}
$$

In particular, it can be seen that this cubic form is positive semidefinite at the threshold and pseudothresholds. 
Using eq. (4), it is easy to derive the following decomposition:

$$
\begin{aligned}
L(4-2 \varepsilon ; 1,1,1) & =\frac{1}{1-2 \varepsilon}\left\{-k^{2} \pi^{-2} L(6-2 \varepsilon ; 2,2,2)\right. \\
& \left.+m_{1}^{2} L(4-2 \varepsilon ; 2,1,1)+m_{2}^{2} L(4-2 \varepsilon ; 1,2,1)+m_{3}^{2} L(4-2 \varepsilon ; 1,1,2)\right\} .
\end{aligned}
$$

This decomposition is similar to one used in [13. Taking into account that

$$
L(6-2 \varepsilon ; 2,2,2)=-\pi^{2} \frac{\partial}{\partial k^{2}} L(4-2 \varepsilon ; 1,1,1)
$$

eq. (17) just reflects the fact that the mass-squared dimension of the integral $L(4-2 \varepsilon ; 1,1,1)$ is $(1-2 \varepsilon)$.

The calculation of each of the integrals on the r.h.s. of (7) is simpler than direct calculation of $L(4-2 \varepsilon ; 1,1,1)$. Using the representation (5), we see that the integrals $L(6-2 \varepsilon ; 2,2,2)$ and $L(4-2 \varepsilon ; 1,1,2)$ are proportional to

$$
\Gamma(2 \varepsilon) \int_{0}^{\infty} \int_{0}^{\infty} \frac{\mathrm{d} \xi \mathrm{d} \eta \xi^{\varepsilon} \eta^{\varepsilon}}{\left(m_{1} \xi+m_{2} \eta+m_{3}\right)^{r-3 \varepsilon}[\text { cubic form }]^{2 \varepsilon}}
$$

where $r=3$ for $L(6-2 \varepsilon ; 2,2,2)$ and $r=2$ for $L(4-2 \varepsilon ; 1,1,2)$.

When $r=3$, the double integral in (9) is convergent, and what we need is just to expand the integrand in $\varepsilon$, keeping the terms of order $\varepsilon$ (since we have got a singular factor $\Gamma(2 \varepsilon)$ in front of the integral). In this way, we get

$$
\begin{array}{r}
\Gamma(2 \varepsilon) \int_{0}^{\infty} \int_{0}^{\infty} \frac{\mathrm{d} \xi \mathrm{d} \eta}{\left(m_{1} \xi+m_{2} \eta+m_{3}\right)^{3}}\left\{1+\varepsilon \ln \xi+\varepsilon \ln \eta+3 \varepsilon \ln \left(m_{1} \xi+m_{2} \eta+m_{3}\right)\right. \\
-2 \varepsilon \ln [\text { cubic form }]\}+\mathcal{O}(\varepsilon) .
\end{array}
$$

When $r=2$, the integral (9) develops a $(1 / \varepsilon)$ singularity as $\xi, \eta \rightarrow \infty$. One can subtract from (9) a simpler integral with the same asymptotic behaviour as $\xi, \eta \rightarrow \infty$,

$$
\Gamma(2 \varepsilon) \int_{0}^{\infty} \int_{0}^{\infty} \frac{\mathrm{d} \xi \mathrm{d} \eta \xi^{\varepsilon} \eta^{\varepsilon}}{\left(m_{1} \xi+m_{2} \eta+m_{3}\right)^{r-3 \varepsilon}\left[\left(m_{1} \xi+m_{2} \eta\right) m_{3} \xi \eta\right]^{2 \varepsilon}},
$$

which can be calculated in terms of $\Gamma$ functions. The difference of (9) and (11) is convergent and yields

$$
\int_{0}^{\infty} \int_{0}^{\infty} \frac{\mathrm{d} \xi \mathrm{d} \eta}{\left(m_{1} \xi+m_{2} \eta+m_{3}\right)^{2}} \ln \left(\frac{\left(m_{1} \xi+m_{2} \eta\right) m_{3} \xi \eta}{[\text { cubic form }]}\right)+\mathcal{O}(\varepsilon) .
$$

When we consider representation (5) for $L(4-2 \varepsilon ; 2,1,1)$ and $L(4-2 \varepsilon ; 1,2,1)$, it does not diverge as $\xi, \eta \rightarrow \infty$. Instead, it does diverge as $\xi \rightarrow 0$ or $\eta \rightarrow 0$. However, these integrals can be reduced to $L(4-2 \varepsilon ; 1,1,2)$ just by a permutation of $m_{1}, m_{2}, m_{3}$ and thus calculated via subtractions similar to (11). 
The most complicated point in calculating the parametric integrals (10) and (12) is how to deal with the contributions involving $\ln [$ cubic form $]$. One of possible ways is to substitute the variables,

$$
\xi=\frac{\sigma}{m_{1}+m_{2}}\left(m_{3}+m_{2} \rho\right), \quad \eta=\frac{\sigma}{m_{1}+m_{2}}\left(m_{3}-m_{1} \rho\right),
$$

and then integrate over $\rho$ between $\left(-m_{3} / m_{2}\right)$ and $\left(m_{3} / m_{1}\right)$. Depending on a region (and whether a pseudothreshold or the threshold is considered), this integral yields an arctangent or a hyperbolic arctangent (the latter can be presented as a logarithm) of an argument involving square roots. The most labour-consuming part was to calculate the remaining integral over $\sigma$, which was done (for the cases considered below) by using some tricky trigonometric substitutions.

\section{General results at the pseudothreshold}

We shall consider the pseudothreshold at $k^{2}=\left(m_{1}+m_{2}-m_{3}\right)^{2}$. The results for the other two pseudothresholds, at $k^{2}=\left(m_{1}-m_{2}+m_{3}\right)^{2}$ and $k^{2}=\left(-m_{1}+m_{2}+m_{3}\right)^{2}$ can be obtained by permutation of the indices $1,2,3$.

It is convenient to introduce the following dilogarithmic functions ${ }^{2}$ :

$$
\begin{aligned}
T^{-}(z) & =\operatorname{Li}_{2}(-z)-\operatorname{Li}_{2}(-1 / z)+\ln z \ln \left((1+z)^{2} / z\right) \\
& =2 \operatorname{Li}_{2}(-z)+\frac{1}{6} \pi^{2}+2 \ln z \ln (1+z)-\frac{1}{2} \ln ^{2} z \\
& =2 \operatorname{Li}_{2}(1 /(1+z))-\frac{1}{6} \pi^{2}+\ln ^{2}(1+z)-\frac{1}{2} \ln ^{2} z \\
T^{+}(z) & =\operatorname{Li}_{2}(1-1 / z)-\mathrm{Li}_{2}(1-z) \\
& =2 \operatorname{Li}_{2}(z)-\frac{1}{3} \pi^{2}+2 \ln z \ln (1-z)-\frac{1}{2} \ln ^{2} z \\
& =2 \operatorname{Li}_{2}(1 /(1-z))+\frac{1}{2} \ln ^{2} z
\end{aligned}
$$

which are antisymmetric under inversion, $T^{ \pm}(1 / z)=-T^{ \pm}(z)$. In particular, $T^{ \pm}(1)=0$ and $T^{ \pm}\left(m_{j} / m_{l}\right)=-T^{ \pm}\left(m_{l} / m_{j}\right)$. Note that the functions $T^{ \pm}(z)$ continue each other to the region of negative $z$ 's, namely $T^{ \pm}(z) \leftrightarrow T^{\mp}(-z)$.

Let us list the results for the integrals involved on the r.h.s. of eq. (7):

$$
\begin{aligned}
& \left.L(6-2 \varepsilon ; 2,2,2)\right|_{k^{2}=\left(m_{1}+m_{2}-m_{3}\right)^{2}}=\pi^{6-2 \varepsilon} \Gamma^{2}(1+\varepsilon) \\
& \times\left\{-\frac{1}{4 \varepsilon} \frac{m_{1}^{1-4 \varepsilon}+m_{2}^{1-4 \varepsilon}-m_{3}^{1-4 \varepsilon}}{m_{1}+m_{2}-m_{3}}-\frac{9}{8}+\frac{m_{1}^{2}+m_{2}^{2}+m_{3}^{2}}{2\left(m_{1}+m_{2}-m_{3}\right)^{2}}\right. \\
& +\frac{m_{1} m_{2} m_{3}}{\left(m_{1}+m_{2}-m_{3}\right)^{3}}\left[\frac{m_{1}-m_{2}}{m_{3}} \ln \frac{m_{1}}{m_{2}}-\frac{m_{2}+m_{3}}{m_{1}} \ln \frac{m_{2}}{m_{3}}-\frac{m_{1}+m_{3}}{m_{2}} \ln \frac{m_{1}}{m_{3}}\right] \\
& +\frac{m_{1}^{2} m_{2}^{2} m_{3}^{2}}{\left(m_{1}+m_{2}-m_{3}\right)^{4}}\left[\frac{m_{1}^{2}-m_{2}^{2}}{m_{1}^{2} m_{2}^{2}} T^{-}\left(\frac{m_{1}}{m_{2}}\right)+\frac{m_{2}^{2}-m_{3}^{2}}{m_{2}^{2} m_{3}^{2}} T^{+}\left(\frac{m_{2}}{m_{3}}\right)+\frac{m_{3}^{2}-m_{1}^{2}}{m_{3}^{2} m_{1}^{2}} T^{+}\left(\frac{m_{3}}{m_{1}}\right)\right.
\end{aligned}
$$

\footnotetext{
${ }^{2} \mathrm{Up}$ to $\ln ^{2} z$ terms, these functions are similar to those used in refs. [4, 11, 12].
} 


$$
\begin{aligned}
& +\left(\frac{1}{m_{3}^{2}}-\frac{1}{2 m_{1}^{2}}-\frac{1}{2 m_{2}^{2}}\right)\left(\ln ^{2} \frac{m_{1}}{m_{2}}+\frac{\pi^{2}}{3}\right) \\
& \left.\left.+\left(\frac{1}{m_{1}^{2}}-\frac{1}{2 m_{2}^{2}}-\frac{1}{2 m_{3}^{2}}\right) \ln ^{2} \frac{m_{2}}{m_{3}}+\left(\frac{1}{m_{2}^{2}}-\frac{1}{2 m_{3}^{2}}-\frac{1}{2 m_{1}^{2}}\right) \ln ^{2} \frac{m_{3}}{m_{1}}\right]\right\}+\mathcal{O}(\varepsilon), \\
& \left.L(4-2 \varepsilon ; 1,1,2)\right|_{k^{2}=\left(m_{1}+m_{2}-m_{3}\right)^{2}}=\pi^{4-2 \varepsilon} \frac{\Gamma^{2}(1+\varepsilon)}{1-\varepsilon} m_{3}^{-4 \varepsilon} \\
& \times\left\{-\frac{1}{2 \varepsilon^{2}}+1+\frac{\pi^{2}}{3}+T^{+}\left(\frac{m_{1}}{m_{3}}\right)+T^{+}\left(\frac{m_{2}}{m_{3}}\right)+\frac{1}{2} \ln ^{2} \frac{m_{1}}{m_{3}}+\frac{1}{2} \ln ^{2} \frac{m_{2}}{m_{3}}\right. \\
& -\frac{2}{m_{1}+m_{2}-m_{3}}\left[m_{1} \ln \frac{m_{1}}{m_{3}}+m_{2} \ln \frac{m_{2}}{m_{3}}\right] \\
& +\frac{1}{\left(m_{1}+m_{2}-m_{3}\right)^{2}}\left[\left(m_{1}^{2}-m_{2}^{2}\right) T^{-}\left(\frac{m_{1}}{m_{2}}\right)-m_{1}^{2} T^{+}\left(\frac{m_{2}}{m_{3}}\right)-m_{2}^{2} T^{+}\left(\frac{m_{1}}{m_{3}}\right)\right. \\
& \left.\left.-\frac{m_{1}^{2}+m_{2}^{2}}{2}\left(\ln ^{2} \frac{m_{1}}{m_{2}}+\frac{\pi^{2}}{3}\right)+\frac{2 m_{2}^{2}-m_{1}^{2}}{2} \ln ^{2} \frac{m_{2}}{m_{3}}+\frac{2 m_{1}^{2}-m_{2}^{2}}{2} \ln ^{2} \frac{m_{1}}{m_{3}}\right]\right\}+\mathcal{O}(\varepsilon), \\
& \left.L(4-2 \varepsilon ; 2,1,1)\right|_{k^{2}=\left(m_{1}+m_{2}-m_{3}\right)^{2}}=\pi^{4-2 \varepsilon} \frac{\Gamma^{2}(1+\varepsilon)}{1-\varepsilon} m_{1}^{-4 \varepsilon} \\
& \times\left\{-\frac{1}{2 \varepsilon^{2}}+1-\frac{\pi^{2}}{6}-T^{-}\left(\frac{m_{1}}{m_{2}}\right)-T^{+}\left(\frac{m_{1}}{m_{3}}\right)+\frac{1}{2} \ln ^{2} \frac{m_{1}}{m_{2}}+\frac{1}{2} \ln ^{2} \frac{m_{1}}{m_{3}}\right. \\
& +\frac{2}{m_{1}+m_{2}-m_{3}}\left[m_{2} \ln \frac{m_{1}}{m_{2}}+m_{3} \ln \frac{m_{3}}{m_{1}}\right] \\
& +\frac{1}{\left(m_{1}+m_{2}-m_{3}\right)^{2}}\left[m_{3}^{2} T^{-}\left(\frac{m_{1}}{m_{2}}\right)+\left(m_{2}^{2}-m_{3}^{2}\right) T^{+}\left(\frac{m_{2}}{m_{3}}\right)+m_{2}^{2} T^{+}\left(\frac{m_{1}}{m_{3}}\right)\right. \\
& \left.\left.+\frac{2 m_{2}^{2}-m_{3}^{2}}{2}\left(\ln ^{2} \frac{m_{1}}{m_{2}}+\frac{\pi^{2}}{3}\right)-\frac{m_{2}^{2}+m_{3}^{2}}{2} \ln ^{2} \frac{m_{2}}{m_{3}}+\frac{2 m_{3}^{2}-m_{2}^{2}}{2} \ln ^{2} \frac{m_{1}}{m_{3}}\right]\right\}+\mathcal{O}(\varepsilon) \text {. }
\end{aligned}
$$

The result for $L(4-2 \varepsilon ; 1,2,1)$ can be obtained from (18) by permutation $m_{1} \leftrightarrow m_{2}$. Substituting eqs. (16)-(18) into eq. (7) we get

$$
\begin{aligned}
& \left.L(4-2 \varepsilon ; 1,1,1)\right|_{k^{2}=\left(m_{1}+m_{2}-m_{3}\right)^{2}}=\pi^{4-2 \varepsilon} \frac{\Gamma^{2}(1+\varepsilon)}{(1-\varepsilon)(1-2 \varepsilon)} \\
& \times\left\{-\frac{1}{2 \varepsilon^{2}}\left(m_{1}^{2-4 \varepsilon}+m_{2}^{2-4 \varepsilon}+m_{3}^{2-4 \varepsilon}\right)+\frac{1}{4 \varepsilon}\left(m_{1}+m_{2}-m_{3}\right)\left(m_{1}^{1-4 \varepsilon}+m_{2}^{1-4 \varepsilon}-m_{3}^{1-4 \varepsilon}\right)\right. \\
& \quad+\frac{7}{8}\left(m_{1}+m_{2}-m_{3}\right)^{2}+\frac{1}{2}\left(m_{1}^{2}+m_{2}^{2}+m_{3}^{2}\right)-\frac{\pi^{2}}{6}\left(m_{1}^{2}+m_{2}^{2}-2 m_{3}^{2}\right) \\
& \quad-\left(m_{1}^{2}-m_{2}^{2}\right) T^{-}\left(\frac{m_{1}}{m_{2}}\right)-\left(m_{2}^{2}-m_{3}^{2}\right) T^{+}\left(\frac{m_{2}}{m_{3}}\right)-\left(m_{3}^{2}-m_{1}^{2}\right) T^{+}\left(\frac{m_{3}}{m_{1}}\right) \\
& \quad+\frac{m_{1}^{2}+m_{2}^{2}}{2} \ln ^{2} \frac{m_{1}}{m_{2}}+\frac{m_{2}^{2}+m_{3}^{2}}{2} \ln ^{2} \frac{m_{2}}{m_{3}}+\frac{m_{3}^{2}+m_{1}^{2}}{2} \ln ^{2} \frac{m_{3}}{m_{1}} \\
& +\frac{m_{1} m_{2} m_{3}}{m_{1}+m_{2}-m_{3}}\left[\frac{m_{1}-m_{2}}{m_{3}} \ln \frac{m_{1}}{m_{2}}-\frac{m_{2}+m_{3}}{m_{1}} \ln \frac{m_{2}}{m_{3}}-\frac{m_{1}+m_{3}}{m_{2}} \ln \frac{m_{1}}{m_{3}}\right]
\end{aligned}
$$




$$
\begin{aligned}
+\frac{m_{1}^{2} m_{2}^{2} m_{3}^{2}}{\left(m_{1}+m_{2}-m_{3}\right)^{2}} & {\left[\frac{m_{1}^{2}-m_{2}^{2}}{m_{1}^{2} m_{2}^{2}} T^{-}\left(\frac{m_{1}}{m_{2}}\right)+\frac{m_{2}^{2}-m_{3}^{2}}{m_{2}^{2} m_{3}^{2}} T^{+}\left(\frac{m_{2}}{m_{3}}\right)+\frac{m_{3}^{2}-m_{1}^{2}}{m_{1}^{2} m_{3}^{2}} T^{+}\left(\frac{m_{3}}{m_{1}}\right)\right.} \\
+ & \left(\frac{1}{m_{3}^{2}}-\frac{1}{2 m_{1}^{2}}-\frac{1}{2 m_{2}^{2}}\right)\left(\ln ^{2} \frac{m_{1}}{m_{2}}+\frac{\pi^{2}}{3}\right) \\
+ & \left.\left.\left(\frac{1}{m_{1}^{2}}-\frac{1}{2 m_{2}^{2}}-\frac{1}{2 m_{3}^{2}}\right) \ln ^{2} \frac{m_{2}}{m_{3}}+\left(\frac{1}{m_{2}^{2}}-\frac{1}{2 m_{3}^{2}}-\frac{1}{2 m_{1}^{2}}\right) \ln ^{2} \frac{m_{1}}{m_{3}}\right]\right\}+\mathcal{O}(\varepsilon)
\end{aligned}
$$

\section{General results at the threshold}

In addition to the notation (14), we shall also introduce the angles $\theta_{1}, \theta_{2}$ and $\theta_{3}$ via

$$
\theta_{i} \equiv \arctan \left(m_{i} \sqrt{\frac{m_{1}+m_{2}+m_{3}}{m_{1} m_{2} m_{3}}}\right) \text {. }
$$

Note that $\theta_{1}+\theta_{2}+\theta_{3}=\pi$. Therefore, they can be undestood as the angles of a certain triangle (cf. e.g. in 25]).

The results for the threshold values of the integrals occurring on the r.h.s. of eq. (7) are

$$
\begin{aligned}
& \left.L(6-2 \varepsilon ; 2,2,2)\right|_{k^{2}=\left(m_{1}+m_{2}+m_{3}\right)^{2}}=\pi^{6-2 \varepsilon} \Gamma^{2}(1+\varepsilon) \\
& \times\left\{-\frac{1}{4 \varepsilon} \frac{m_{1}^{1-4 \varepsilon}+m_{2}^{1-4 \varepsilon}+m_{3}^{1-4 \varepsilon}}{m_{1}+m_{2}+m_{3}}-\frac{9}{8}+\frac{m_{1}^{2}+m_{2}^{2}+m_{3}^{2}}{2\left(m_{1}+m_{2}+m_{3}\right)^{2}}\right. \\
& +\frac{m_{1} m_{2} m_{3}}{\left(m_{1}+m_{2}+m_{3}\right)^{3}}\left[\frac{m_{1}-m_{2}}{m_{3}} \ln \frac{m_{1}}{m_{2}}+\frac{m_{2}-m_{3}}{m_{1}} \ln \frac{m_{2}}{m_{3}}+\frac{m_{3}-m_{1}}{m_{2}} \ln \frac{m_{3}}{m_{1}}\right] \\
& +\frac{m_{1}^{2} m_{2}^{2} m_{3}^{2}}{\left(m_{1}+m_{2}+m_{3}\right)^{4}}\left[\frac{m_{1}^{2}-m_{2}^{2}}{m_{1}^{2} m_{2}^{2}} T^{-}\left(\frac{m_{1}}{m_{2}}\right)+\frac{m_{2}^{2}-m_{3}^{2}}{m_{2}^{2} m_{3}^{2}} T^{-}\left(\frac{m_{2}}{m_{3}}\right)+\frac{m_{3}^{2}-m_{1}^{2}}{m_{3}^{2} m_{1}^{2}} T^{-}\left(\frac{m_{3}}{m_{1}}\right)\right. \\
& +\left(\frac{1}{m_{3}^{2}}-\frac{1}{2 m_{1}^{2}}-\frac{1}{2 m_{2}^{2}}\right) \ln ^{2} \frac{m_{1}}{m_{2}}+\left(\frac{1}{m_{1}^{2}}-\frac{1}{2 m_{2}^{2}}-\frac{1}{2 m_{3}^{2}}\right) \ln ^{2} \frac{m_{2}}{m_{3}} \\
& +\left(\frac{1}{m_{2}^{2}}-\frac{1}{2 m_{3}^{2}}-\frac{1}{2 m_{1}^{2}}\right) \ln ^{2} \frac{m_{3}}{m_{1}}+\frac{4 \pi^{2}}{3}\left(\frac{1}{m_{1}^{2}}+\frac{1}{m_{2}^{2}}+\frac{1}{m_{3}^{2}}\right) \\
& \left.\left.-4 \pi\left(\frac{\theta_{1}}{m_{1}^{2}}+\frac{\theta_{2}}{m_{2}^{2}}+\frac{\theta_{3}}{m_{3}^{2}}\right)+4 \pi\left(\frac{1}{m_{1}}+\frac{1}{m_{2}}+\frac{1}{m_{3}}\right) \sqrt{\frac{m_{1}+m_{2}+m_{3}}{m_{1} m_{2} m_{3}}}\right]\right\}+\mathcal{O}(\varepsilon), \\
& \left.L(4-2 \varepsilon ; 1,1,2)\right|_{k^{2}=\left(m_{1}+m_{2}+m_{3}\right)^{2}}=\pi^{4-2 \varepsilon} \frac{\Gamma^{2}(1+\varepsilon)}{1-\varepsilon} m_{3}^{-4 \varepsilon} \\
& \times\left\{-\frac{1}{2 \varepsilon^{2}}+1+\frac{4 \pi^{2}}{3}-4 \pi \theta_{3}+T^{-}\left(\frac{m_{1}}{m_{3}}\right)+T^{-}\left(\frac{m_{2}}{m_{3}}\right)+\frac{1}{2} \ln ^{2} \frac{m_{1}}{m_{3}}+\frac{1}{2} \ln ^{2} \frac{m_{2}}{m_{3}}\right. \\
& -\frac{2}{m_{1}+m_{2}+m_{3}}\left[m_{1} \ln \frac{m_{1}}{m_{3}}+m_{2} \ln \frac{m_{2}}{m_{3}}\right] \\
& +\frac{1}{\left(m_{1}+m_{2}+m_{3}\right)^{2}}\left[\left(m_{1}^{2}-m_{2}^{2}\right) T^{-}\left(\frac{m_{1}}{m_{2}}\right)-m_{1}^{2} T^{-}\left(\frac{m_{2}}{m_{3}}\right)-m_{2}^{2} T^{-}\left(\frac{m_{1}}{m_{3}}\right)\right.
\end{aligned}
$$




$$
\begin{gathered}
-\frac{m_{1}^{2}+m_{2}^{2}}{2} \ln ^{2} \frac{m_{1}}{m_{2}}+\frac{2 m_{1}^{2}-m_{2}^{2}}{2} \ln ^{2} \frac{m_{1}}{m_{3}}+\frac{2 m_{2}^{2}-m_{1}^{2}}{2} \ln ^{2} \frac{m_{2}}{m_{3}} \\
\left.\left.+\frac{4 \pi^{2}}{3}\left(m_{1}^{2}+m_{2}^{2}\right)-4 \pi\left(m_{2}^{2} \theta_{1}+m_{1}^{2} \theta_{2}\right)-4 \pi \sqrt{m_{1} m_{2} m_{3}\left(m_{1}+m_{2}+m_{3}\right)}\right]\right\}+\mathcal{O}(\varepsilon) .
\end{gathered}
$$

The results for $L(4-2 \varepsilon ; 2,1,1)$ and $L(4-2 \varepsilon ; 1,2,1)$ can be obtained from (22) by permutations $m_{3} \leftrightarrow m_{1}$ and $m_{3} \leftrightarrow m_{2}$, respectively. Using eq. (7), we get

$$
\begin{aligned}
& \left.L(4-2 \varepsilon ; 1,1,1)\right|_{k^{2}=\left(m_{1}+m_{2}+m_{3}\right)^{2}}=\pi^{4-2 \varepsilon} \frac{\Gamma^{2}(1+\varepsilon)}{(1-\varepsilon)(1-2 \varepsilon)} \\
& \times\left\{-\frac{1}{2 \varepsilon^{2}}\left(m_{1}^{2-4 \varepsilon}+m_{2}^{2-4 \varepsilon}+m_{3}^{2-4 \varepsilon}\right)+\frac{1}{4 \varepsilon}\left(m_{1}+m_{2}+m_{3}\right)\left(m_{1}^{1-4 \varepsilon}+m_{2}^{1-4 \varepsilon}+m_{3}^{1-4 \varepsilon}\right)\right. \\
& +\frac{7}{8}\left(m_{1}+m_{2}+m_{3}\right)^{2}+\left(\frac{1}{2}+\frac{4 \pi^{2}}{3}\right)\left(m_{1}^{2}+m_{2}^{2}+m_{3}^{2}\right)-4 \pi\left(m_{1}^{2} \theta_{1}+m_{2}^{2} \theta_{2}+m_{3}^{2} \theta_{3}\right) \\
& -\left(m_{1}^{2}-m_{2}^{2}\right) T^{-}\left(\frac{m_{1}}{m_{2}}\right)-\left(m_{2}^{2}-m_{3}^{2}\right) T^{-}\left(\frac{m_{2}}{m_{3}}\right)-\left(m_{3}^{2}-m_{1}^{2}\right) T^{-}\left(\frac{m_{3}}{m_{1}}\right) \\
& +\frac{m_{1}^{2}+m_{2}^{2}}{2} \ln ^{2} \frac{m_{1}}{m_{2}}+\frac{m_{2}^{2}+m_{3}^{2}}{2} \ln ^{2} \frac{m_{2}}{m_{3}}+\frac{m_{3}^{2}+m_{1}^{2}}{2} \ln ^{2} \frac{m_{3}}{m_{1}}-4 \pi \sqrt{m_{1} m_{2} m_{3}\left(m_{1}+m_{2}+m_{3}\right)} \\
& +\frac{m_{1} m_{2} m_{3}}{m_{1}+m_{2}+m_{3}}\left[\frac{m_{1}-m_{2}}{m_{3}} \ln \frac{m_{1}}{m_{2}}+\frac{m_{2}-m_{3}}{m_{1}} \ln \frac{m_{2}}{m_{3}}+\frac{m_{3}-m_{1}}{m_{2}} \ln \frac{m_{3}}{m_{1}}\right] \\
& +\frac{m_{1}^{2} m_{2}^{2} m_{3}^{2}}{\left(m_{1}+m_{2}+m_{3}\right)^{2}}\left[\frac{m_{1}^{2}-m_{2}^{2}}{m_{1}^{2} m_{2}^{2}} T^{-}\left(\frac{m_{1}}{m_{2}}\right)+\frac{m_{2}^{2}-m_{3}^{2}}{m_{2}^{2} m_{3}^{2}} T^{-}\left(\frac{m_{2}}{m_{3}}\right)+\frac{m_{3}^{2}-m_{1}^{2}}{m_{1}^{2} m_{3}^{2}} T^{-}\left(\frac{m_{3}}{m_{1}}\right)\right. \\
& +\left(\frac{1}{m_{3}^{2}}-\frac{1}{2 m_{1}^{2}}-\frac{1}{2 m_{2}^{2}}\right) \ln ^{2} \frac{m_{1}}{m_{2}}+\left(\frac{1}{m_{1}^{2}}-\frac{1}{2 m_{2}^{2}}-\frac{1}{2 m_{3}^{2}}\right) \ln ^{2} \frac{m_{2}}{m_{3}} \\
& +\left(\frac{1}{m_{2}^{2}}-\frac{1}{2 m_{3}^{2}}-\frac{1}{2 m_{1}^{2}}\right) \ln ^{2} \frac{m_{1}}{m_{3}}+\frac{4 \pi^{2}}{3}\left(\frac{1}{m_{1}^{2}}+\frac{1}{m_{2}^{2}}+\frac{1}{m_{3}^{2}}\right) \\
& \left.\left.-4 \pi\left(\frac{\theta_{1}}{m_{1}^{2}}+\frac{\theta_{2}}{m_{2}^{2}}+\frac{\theta_{3}}{m_{3}^{2}}\right)+4 \pi\left(\frac{1}{m_{1}}+\frac{1}{m_{2}}+\frac{1}{m_{3}}\right) \sqrt{\frac{m_{1}+m_{2}+m_{3}}{m_{1} m_{2} m_{3}}}\right]\right\}+\mathcal{O}(\varepsilon)(23)
\end{aligned}
$$

The presented expressions explicitly obey all required symmetries. In particular, the result (22) is symmetric under $m_{1} \leftrightarrow m_{2}$, whereas eqs. (21) and (23) are totally symmetric in $m_{1}, m_{2}, m_{3}$.

\section{Some special cases}

When two of the masses are equal (say, $m_{1}=m_{2} \equiv m, m_{3} \equiv M$ ) the presented general expressions can be simplified. Let us consider, as an example, the integral $L(4-2 \varepsilon ; 1,1,1)$ and introduce a dimensionless variable $\mu \equiv M / m$.

At two different pseudothresholds, $k^{2}=(2 m-M)^{2}=(2-\mu)^{2} m^{2}$ and $k^{2}=M^{2}=\mu^{2} m^{2}$, we get the following results:

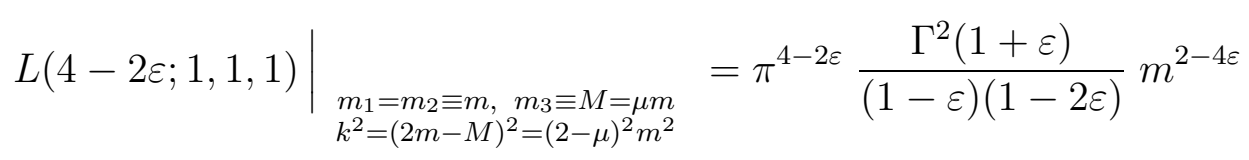




$$
\begin{gathered}
\times\left\{-\frac{2+\mu^{2}}{2 \varepsilon^{2}}+\frac{(2-\mu)^{2}}{4 \varepsilon}+\frac{2 \mu^{2}}{\varepsilon} \ln \mu+\frac{1}{2}\left(2+\mu^{2}\right)+\frac{7}{8}(2-\mu)^{2}+\frac{6-2 \mu+\mu^{2}}{2-\mu} \mu \ln \mu\right. \\
\left.-2 \mu^{2} \ln ^{2} \mu-\frac{4(1-\mu)^{2}(1+\mu)(3-\mu)}{(2-\mu)^{2}}\left[\operatorname{Li}_{2}(1-\mu)+\frac{\pi^{2}}{12}\right]\right\}+\mathcal{O}(\varepsilon), \\
L(4-2 \varepsilon ; 1,1,1) \mid \begin{array}{c}
m_{1}=m_{2} \equiv m, m_{3} \equiv M=\mu m \\
k^{2}=M^{2}=\mu^{2} m^{2}
\end{array}=\pi^{4-2 \varepsilon} \frac{\Gamma^{2}(1+\varepsilon)}{(1-\varepsilon)(1-2 \varepsilon)} m^{2-4 \varepsilon} \\
\times\left\{\begin{array}{c}
2+\mu^{2} \\
2 \varepsilon^{2} \\
-\frac{\mu^{2}}{4 \varepsilon}+\frac{2}{\varepsilon} \mu^{2} \ln \mu+1+\frac{11}{8} \mu^{2}-\left(2+\mu^{2}\right) \ln \mu-2 \mu^{2} \ln ^{2} \mu \\
\left.+\frac{\left(1-\mu^{2}\right)^{2}}{\mu^{2}}\left[\operatorname{Li}_{2}\left(1-\mu^{2}\right)-\frac{\pi^{2}}{6}\right]\right\}+\mathcal{O}(\varepsilon) .
\end{array}\right.
\end{gathered}
$$

Note that the latter expression is relevant for the on-shell calculations and corresponds to the results presented in refs. [11, 12].

At the threshold, when $k^{2}=(2 m+M)^{2}=(2+\mu)^{2} m^{2}$, we get

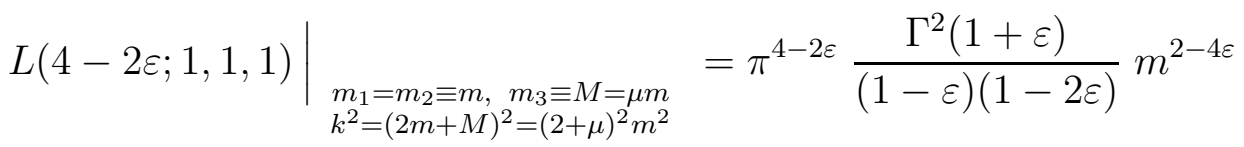

$$
\begin{aligned}
& \times\left\{-\frac{2+\mu^{2}}{2 \varepsilon^{2}}+\frac{(2+\mu)^{2}}{4 \varepsilon}+\frac{2 \mu^{2}}{\varepsilon} \ln \mu+\frac{1}{2}\left(2+\mu^{2}\right)+\frac{7}{8}(2+\mu)^{2}\right. \\
& -\frac{6+2 \mu+\mu^{2}}{2+\mu} \mu \ln \mu-2 \mu^{2} \ln ^{2} \mu-\frac{4 \mu^{1 / 2}}{(2+\mu)^{3 / 2}}\left(3+2 \mu+\mu^{2}\right) \pi \\
& \left.+\frac{4(1+\mu)^{2}(1-\mu)(3+\mu)}{(2+\mu)^{2}}\left[\operatorname{Li}_{2}\left(\frac{1}{1+\mu}\right)+\frac{1}{2} \ln ^{2}(1+\mu)+\pi \arccos \left(\frac{1}{1+\mu}\right)-\frac{5 \pi^{2}}{12}\right]\right\}+\mathcal{O}(\varepsilon) \text {. }
\end{aligned}
$$

For $\mu=1$, i.e. when all masses are equal, the results (24) and (25) give the same as eq. (30) of the paper [5] (see also in [4]),

$$
\left.L(4-2 \varepsilon ; 1,1,1)\right|_{\substack{m_{1}=m_{2}=m_{3}=m \\ k^{2}=m^{2}}}=\pi^{4-2 \varepsilon} \frac{\Gamma^{2}(1+\varepsilon)}{(1-\varepsilon)(1-2 \varepsilon)} m^{2-4 \varepsilon}\left\{-\frac{3}{2 \varepsilon^{2}}+\frac{1}{4 \varepsilon}+\frac{19}{8}\right\}+\mathcal{O}(\varepsilon),
$$

whereas eq. (26) yields the threshold value of the sunset integral with equal masses

$$
\left.L(4-2 \varepsilon ; 1,1,1)\right|_{\substack{m_{1}=m_{2}=m_{3}=m \\ k^{2}=9 m^{2}}}=\pi^{4-2 \varepsilon} \frac{\Gamma^{2}(1+\varepsilon)}{(1-\varepsilon)(1-2 \varepsilon)} m^{2-4 \varepsilon}\left\{-\frac{3}{2 \varepsilon^{2}}+\frac{9}{4 \varepsilon}+\frac{75}{8}-\frac{8 \pi}{\sqrt{3}}\right\}+\mathcal{O}(\varepsilon) .
$$

We have also reproduced the same result (28) by using the dispersion technique.

\section{Conclusions}

We have obtained analytic results for the threshold and pseudothreshold values of the sunset diagram with arbitrary masses. The results are expressed in terms of dilogarithms 
of ratios of the masses, all other functions being elementary. The results are explicitly symmetric with respect to all symmetries they have to obey. Certain checks, including numerical ones, confirm that the results are self-consistent. Note that most of the terms in eqs. (21)-(23) look just as naive analytic continuation of the results (16)-(19), if one changes $m_{3}$ into $\left(-m_{3}\right)$. The non-trivial terms are those involving $\pi$, including the $\theta_{i}$ terms.

We note that the complete (unsubtracted) results, involving the $1 / \varepsilon^{2}$ and $1 / \varepsilon$ ultraviolet poles, are simpler than the finite function obtained by subtraction of two terms of the expansion in $k^{2}$ near $k^{2}=0$ (cf. e.g. in [15]). In the latter case, we would also get dilogarithms (or Clausen functions) corresponding to the vacuum diagrams [26, 16]. In particular, for the equal-mass case (28) the $\mathrm{Cl}_{2}(\pi / 3)$ contribution is missing in eq. (28).

As an extension of the presented approach, two-loop self-energy diagrams with four and five propagators may also be considered. For example, the exact result for the pseudothreshold value of the "master" diagram with equal masses was presented in [27]. Although the results obtained may be used for all purposes listed in the introduction, we would like to emphasize that their application to constructing expansions near the thresholds is one of the most important points.

Acknowledgements. We would like to thank A.G. Grozin, V.A. Smirnov and J.B. Tausk for useful discussions and help in checking results for some special cases. A. D. and N. U. are grateful to the Instituut-Lorentz, University of Leiden for their hospitality during our visits. The research was mainly supported by the EU grant INTAS-93-0744. A partial support from the RFBR grants 95-02-05794 (N. U.) and 96-01-00654 (A. D.) is acknowledged.

\section{References}

[1] G. 't Hooft and M. Veltman, Nucl.Phys. B44 (1972) 189;

C.G. Bollini and J.J. Giambiagi, Nuovo Cim. 12B (1972) 20.

[2] G. Weiglein, R. Scharf and M. Böhm, Nucl. Phys. B416 (1994) 606;

O.V. Tarasov, Nucl. Phys. B502 (1997) 455;

A. Ghinculov and Y.-P. Yao, Freiburg preprint THEP-97-04 (hep-ph/9702266).

[3] G. Källén and A. Sabry, Dan. Mat. Fys. Medd. 29, No.17 (1955) 1;

D.J. Broadhurst, Phys. Lett. B101 (1981) 423;

T.H. Chang, K.J.F. Gaemers and W.L. van Neerven, Nucl. Phys. B202 (1982) 407;

A. Djouadi, Nuovo Cim. 100A (1988) 357;

B.A. Kniehl, Nucl. Phys. B347 (1990) 86. A.V. Kotikov, Phys. Lett. B254 (1991) 158; Mod. Phys. Lett. A6 (1991) 677;

P.N. Maher, L. Durand and K. Riesselmann, Phys. Rev. D48 (1993) 1061;

D.T. Gegelia, K.Sh. Japaridze and K.Sh. Turashvili, Teor. Mat. Fiz. 101 (1994) 225.

[4] D.J. Broadhurst, Z. Phys. C47 (1990) 115.

[5] D.J. Broadhurst, J. Fleischer and O.V. Tarasov, Z. Phys. C60 (1993) 287. 
[6] R. Scharf, Diploma thesis (Würzburg, 1991); Doctoral thesis (Würzburg, 1994);

R. Scharf and J.B. Tausk, Nucl. Phys. B412 (1994) 523.

[7] A.K. Rajantie, Nucl. Phys. B480 (1996) 729.

[8] F.A. Berends, A.I. Davydychev and V.A. Smirnov, Nucl. Phys. B478 (1996) 59.

[9] F.V. Tkachov, e-print hep-ph/9703424.

[10] M. Beneke and V.A. Smirnov, Preprint CERN-TH-97-315 (hep-ph/9711391)

[11] D.J. Broadhurst and A.G. Grozin, Phys. Rev. D52 (1995) 4082.

[12] A. Czarnecki and K. Melnikov, Karlsruhe preprint TTP-97-08 (hep-ph/9703277).

[13] A. Ghinculov and J.J. van der Bij, Nucl. Phys. B436 (1995) 30.

[14] D. Kreimer, Phys. Lett. B273 (1991) 277;

J. Fujimoto, Y. Shimizu, K. Kato and Y. Oyanagi, KEK preprint 92-213;

F.A. Berends and J.B. Tausk, Nucl. Phys. B421 (1994) 456;

F.A. Lunev, Phys. Rev. D50 (1994) 7735;

A. Czarnecki, U. Kilian and D. Kreimer, Nucl. Phys. B433 (1995) 259;

S. Bauberger, F.A. Berends, M. Böhm and M. Buza, Nucl.Phys. B434 (1995) 383;

S. Bauberger and M. Böhm, Nucl. Phys. B445 (1995) 25.

[15] P. Post and J.B. Tausk, Mod. Phys. Lett. A11 (1996) 2115.

[16] A.I. Davydychev and J.B. Tausk, Nucl. Phys. B397 (1993) 123.

[17] J. Fleischer and O. V. Tarasov, Z. Phys. C64 (1994) 413.

[18] O.V. Tarasov, Nucl. Phys. B480 (1996) 397.

[19] A.I. Davydychev, V.A. Smirnov and J.B. Tausk, Nucl. Phys. B410 (1993) 325.

[20] F.A. Berends, M. Buza, M. Böhm and R. Scharf, Z. Phys. C63 (1994) 227.

[21] E. Mendels, Nuovo Cim. 45A (1978) 87.

[22] A.I. Davydychev and J.B. Tausk, Phys. Rev. D53 (1996) 7381.

[23] H. Cheng and T.T. Wu, Expanding protons: scattering at high energies (MIT press, Cambridge, Massachusetts, 1987).

[24] K.S. Bjørkevoll, G. Fäldt and P. Osland, Nucl. Phys. B386 (1992) 303.

[25] A.I. Davydychev and R. Delbourgo, Preprint UTAS-PHYS-97-12 (hep-th/9709216).

[26] J.J. van der Bij and M. Veltman, Nucl. Phys. B231 (1984) 205;

F. Hoogeveen, Nucl. Phys. B259 (1985) 19;

J.J. van der Bij and F. Hoogeveen, Nucl. Phys. B283 (1987) 477;

C. Ford, I. Jack and D.R.T. Jones, Nucl.Phys. B387 (1992) 373.

[27] V. Borodulin and G. Jikia, Phys. Lett. B391 (1997) 434. 